\title{
Intratumoral and peritumoral lymphocytic responses correlate with survival in rectal cancer
}

\author{
Che-Wei Su${ }^{1}$, Tzu-Yin Tang ${ }^{2}$, Chi-Jung Li $^{1}$, Yu-Chuen Huang ${ }^{3,4}$, Yu-Jen Chen ${ }^{1,4,5}$ \\ ${ }^{1}$ Department of Radiation Oncology, MacKay Memorial Hospital, Taipei, Taiwan; ${ }^{2}$ Department of Pathology, MacKay Memorial Hospital, Taipei, \\ Taiwan; ${ }^{3}$ School of Chinese Medicine, China Medical University, Taichung, Taiwan; ${ }^{4}$ Department of Medical Research, China Medical University \\ Hospital, Taichung; ${ }^{5}$ Department of Nursing, MacKay Junior College of Medicine, Nursing, and Management, Taipei, Taiwan \\ Contributions: (I) Conception and design: YJ Chen; (II) Administrative support: CW Su, TY Tang, CJ Li; (III) Provision of study materials or patients: \\ TY Tang, YJ Chen; (IV) Collection and assembly of data: CJ Li; (V) Data analysis and interpretation: CW Su, YC Huang; (VI) Manuscript writing: \\ All authors; (VII) Final approval of manuscript: All authors. \\ Correspondence to: Yu-Jen Chen. No. 45, Minsheng Rd., Tamsui District, New Taipei City 25160, Taiwan. Email: chenmdphd@gmail.com; Yu-Chuen \\ Huang. No. 91, Hsueh-Shih Road, Taichung 404, Taiwan. Email: yuchuen@mail.cmu.edu.tw.
}

Background: Colorectal cancer (CRC) with high level of microsatellite instability (MSI-H) is associated with improved survival. Histopathological assessment of prominent infiltration of lymphocytes in tumor microenvironment (TME), including intratumoral lymphocytic response (ILR) and peritumoral lymphocytic response (PLR), was utilized to predict MSI-H. However, the direct pathological evidence of lymphocytic response predicting survival of rectal cancer is lacking due to the predominant neoadjuvant concurrent chemoradiotherapy (CCRT) treatment. This study aims to identify whether the phenotype of PLR and ILR is associated with the clinical outcome of locally-advanced rectal cancer receiving definitive surgery followed by adjuvant CCRT.

Methods: From 2005 to 2018, among the 121 patients enrolled from MacKay Memorial Hospital, 55 specimen was assessable for lymphocytic response. ILR and PLR were assessed according to the cancer reporting protocol released by the College of American Pathologists (CAP). Based on positive or negative ILR/PLR, we categorized each patient as one of the four groups: ILR+/PLR+, ILR+/PLR-, ILR-/PLR+, or ILR-/PLR-.

Results: ILR-/PLR- was significantly associated with poorer overall survival, compared to either positive lymphocytic response of ILR or PLR. Multivariate analysis revealed ILR-/PLR- as a significant risk factor for overall survival after adjusting with clinical characteristics.

Conclusions: Lymphocytic response in tumor microenvironment (TME) can be a predictor for poor survival outcome and a potential indicator for immunotherapy.

Keywords: Rectal cancer; tumor microenvironment; lymphocytic response; immunotherapy

Received: 14 June 2021; Accepted: 26 July 2021; Published: 30 September 2021.

doi: $10.21037 /$ tro-21-13

View this article at: https://dx.doi.org/10.21037/tro-21-13

\section{Introduction}

Colorectal cancer (CRC) is the third most frequently diagnosed cancer worldwide (1). Studies have revealed the presence of microsatellite instability (MSI) as a hallmark of prognosis in CRC $(2,3)$. Tumors showing high level of microsatellite instability (MSI-H) have improved survival compared with low level of microsatellite instability (MSI-L) or microsatellite stable (MSS) (4). MSI-H has been associated with the prominent infiltration of immune cells, which is also indicated as the cardinal role in orchestrating the response to immune therapy within tumor 
microenvironment (TME) (5). The prognostic benefit may be attributed to DNA mismatch repair deficiency causing frameshift mutations which results in the introduction of antigen and immune cell infiltration. TME comprises intratumoral lymphocytic response (ILR) reflecting the infiltration of T-lymphocytes within the tumor, and peritumoral lymphocytic response (PLR) reflecting the invasive margin of tumor (6). Therefore, the pattern of lymphocytic infiltration evaluated by ILR and PLR is a potentially reliable clinical indicator.

Immune tumor subtypes can be categorized as immune desert, excluded, and inflamed, reflecting the infiltrating pattern of T-lymphocytes (7). Immune inflamed tumors are usually infiltrated with T-lymphocytes. Immune desert tumors are characterized with the absence of T-lymphocyte infiltration. Immune excluded tumors retain T-lymphocytes at the invasive margin but no T-lymphocyte in tumor bed.

In a large cohort study for 2,369 CRC patients, Rozek et al. reported the possible impact of tumor-infiltrating lymphocytes (TIL, as known as ILR) and Crohn's-like lymphoid reaction (CLR, as known as PLR) on CRCspecific and overall survival (8). Notably, only TIL but not CLR had significant impact on cancer-specific and overall survival in rectal cancer patients. Historically, colon cancer and rectal cancer are usually analyzed together. However, the treatment paradigm is different between these two entities. In colon cancer, the definitive treatment is surgery as possible, even for locally-advanced stage. On the other hand, it is common to give neoadjuvant chemoradiotherapy (CCRT) before surgery in rectal cancer with invasion through muscularis propria into pericolorectal tissue or clinical positive lymph node (9). The disadvantage of surgery following neoadjuvant CCRT is the complete pathological staging can only be acquired after treatment. The initial specimen is derived from biopsy only, resulting in the plight of interpretation of ILR and PLR, which generally require larger size of specimen. Taken together, the precisely pathological evidence of lymphocyte infiltration in rectal cancer is still lacking.

In this study, we collect locally-advanced rectal cancer cases that underwent surgery with adjuvant CCRT only. There was no neoadjuvant therapy, so that ILR and PLR could be examined with complete specimen. The aim is to identify whether the phenotype of PLR and ILR is associated with the clinical outcome of rectal cancer. We present the following article in accordance with the STROBE reporting checklist (available at https://dx.doi. org/10.21037/tro-21-13).

\section{Methods}

\section{Patients}

This retrospective study enrolled 121 patients from MacKay Memorial Hospital (Taipei, Taiwan) between September 2005 and March 2018. The inclusion criteria were pathologically confirmed rectal cancer, staging at least $\geq \mathrm{T} 3$ or $\geq \mathrm{N} 1$, and treated with definitive surgery, followed by adjuvant CCRT. Complete specimen were reviewed by experienced pathologists to identify ILR and PLR. Specimen that is not assessable for lymphocytic response were excluded $(n=66)$. The study was conducted in accordance with the Declaration of Helsinki (as revised in 2013). This study was approved by the local institutional review board of MacKay Memorial Hospital (IRB number: 20MMHIS005e; Date of decision: February 04, 2020). Informed consent was waived because this retrospective study used only pre-existing medical data.

\section{Lymphocytic response assessment}

Lymphocytic response, including intratumoral lymphocytic response (ILR) and peritumoral lymphocytic response (PLR), was assessed according to the cancer reporting protocol released by the College of American Pathologists (Figure 1). The ILR was graded as three levels: none (no lymphocyte), mild to moderate (1 or 2 lymphocytes per $400 \times$ high-power field), and marked (3 or more lymphocytes per high-power field). PLR, also known as Crohn-like response, is defined as the presence of lymphoid aggregates or follicles at the tumor edge, not associated with preexisting lymph node (10). It was also graded as none, mild to moderate, and marked.

If the lymphocytic response is graded as none, it would be identified as negative for ILR or PLR. Otherwise, it would be identified as positive for ILR or PLR, no matter the response was mild-to-moderate or marked. Based on positive or negative ILR/PLR, we categorized each patient as one of the four groups: ILR+/PLR+, ILR+/PLR-, ILR-/ PLR+, or ILR-/PLR-.

\section{Treatment and outcome}

All eligible patients were treated with definitive operation, and because all patients meets the criteria of $\geq \mathrm{pT} 3$ or $\geq \mathrm{pN} 1$, they received adjuvant CCRT. Surgical intervention is one of the following: (I) radical proctectomy (II) abdominal perineal resection (III) laparoscopic low anterior resection 

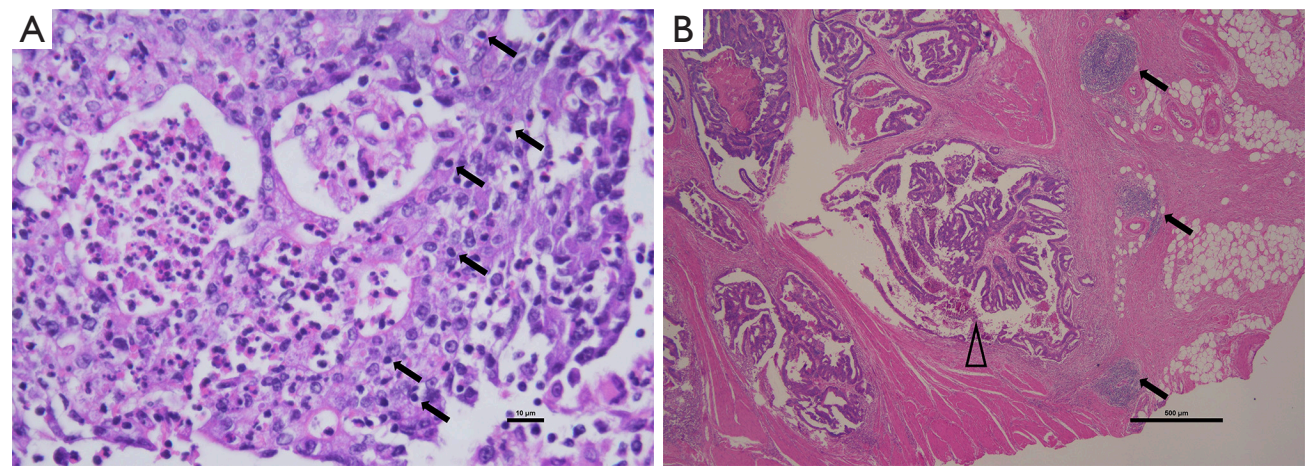

Figure 1 Histopathology of intratumoral lymphocytic response (ILR) and peritumoral lymphocytic response (PLR). (A) ILR. Lymphocytes are present in the neoplastic epithelium (arrows) (Hematoxylin and eosin stain, 400x); (B) PLR (arrows) manifests as lymphoid aggregates peripheral to tumor (open arrow head) (Hematoxylin and eosin stain, 20x).

(IV) local excision. Adjuvant CCRT regimen is 45-60 Gy (median 54 Gy; maximum dose $<107 \%$ of prescription dose) to planning target volume (PTV) including surgical bed and lymphatic area at risk, with safety margin of 5-7 $\mathrm{mm}$. All patients received intensity-modulated radiotherapy (IMRT) technique. Concurrent chemotherapy regimen was one of the following: (I) oral UFUR (tegafur/ uracil) (II) oral capecitabine (III) intravenous infusion fluorouracil/leucovorin (Table 1).

The primary endpoint of this study was overall survival (OS), and secondary endpoints included progression-free survival (PFS), local-recurrence-free survival (LRFS), and distantmetastasis-free survival (DMFS). Survival time was calculated as the time from diagnosis to death, disease progression, locoregional recurrence, or distant metastasis, respectively.

\section{Statistical analysis}

Survival outcomes of the high- and low-risk groups were compared with log-rank tests on total population $(n=55)$ of this study. Uni- and multivariate analysis was performed using Cox regression to examine the predictive potential of ILR and PLR compared with traditional clinical variables. The statistical analysis was performed by SPSS (IBM Corp. Released 2019. IBM SPSS Statistics for Macintosh, Version 26.0. Armonk, NY: IBM Corp).

\section{Results}

\section{Patients and treatment characteristics}

A total of 55 patients of locally-advanced rectal cancer who underwent definitive surgery followed by adjuvant CCRT were subjected to analysis. The median follow-up time was 32.2 (IQR 12.9-58.5) months. The clinical characteristics of the cohorts are summarized in Table 1 .

\section{Survival analysis}

The Kaplan-Meier survival curve for OS comparing ILR-/ PLR- with either positive response for ILR or PLR were presented in Figure 2.

\section{Integration with clinical characteristics}

Univariate and multivariate analyses were performed to compare the predictive ability of lymphocytic response on OS with traditional clinical variables, including age, clinical stage, and the margin status. In univariate analysis, lymphocytic response had no significant hazard ratio (HR) for OS between the four groups. In multivariate analysis, both ILR-/PLR- and pathological lymph node stage were significant risk factors for OS. The detailed results were presented in Table 2.

\section{Discussion}

In this study, we investigated the role of intratumoral and peritumoral lymphocytic response in the prognosis of locally-advanced rectal cancers treated with definitive surgery followed by adjuvant CCRT. We found the tumors with negative neither intratumoral nor peritumoral lymphocytic response were significantly correlated with poorer survival outcome, comparing to tumors with positive 
Table 1 Clinical characteristics

\begin{tabular}{|c|c|}
\hline Characteristic & Patients $(n=121)$ \\
\hline Age (year) & $58.16 \pm 11.64$ \\
\hline \multicolumn{2}{|l|}{ Gender } \\
\hline Male & $74(61.2)$ \\
\hline Female & $47(38.8)$ \\
\hline \multicolumn{2}{|c|}{ ECOG performance status } \\
\hline 0 & $104(86)$ \\
\hline 1 & $15(12.4)$ \\
\hline 2 & $2(1.7)$ \\
\hline \multicolumn{2}{|l|}{ pT } \\
\hline pT1 & $4(3.3)$ \\
\hline рT2 & $13(10.7)$ \\
\hline рT3 & $99(81.8)$ \\
\hline pT4 & $5(4.1)$ \\
\hline \multicolumn{2}{|l|}{$\mathrm{pN}$} \\
\hline pNO & $3(2.5)$ \\
\hline pN1 & $53(43.8)$ \\
\hline pN2 & $65(53.7)$ \\
\hline \multicolumn{2}{|l|}{ Pathological stage } \\
\hline II & $3(2.5)$ \\
\hline III & $116(95.9)$ \\
\hline IV & $2(1.7)$ \\
\hline \multicolumn{2}{|l|}{ ECE } \\
\hline Positive & $18(14.9)$ \\
\hline Negative & $30(24.8)$ \\
\hline Missing & $73(60.3)$ \\
\hline \multicolumn{2}{|c|}{ Lymphocytic response } \\
\hline ILR+/PLR+ & $23(19)$ \\
\hline ILR+/PLR- & $2(1.7)$ \\
\hline ILR-/PLR+ & $15(12.4)$ \\
\hline ILR-/PLR- & $15(12.4)$ \\
\hline Not assessable & $66(54.5)$ \\
\hline \multicolumn{2}{|l|}{ Surgical margin } \\
\hline Positive margin & $19(15.7)$ \\
\hline$\leq 5 \mathrm{~mm}$ & $49(40.5)$ \\
\hline$>5 \mathrm{~mm}$ & $53(43.8)$ \\
\hline
\end{tabular}

Table 1 (continued)
Table 1 (continued)

\begin{tabular}{lc}
\hline Characteristic & Patients $(\mathrm{n}=121)$ \\
\hline Chemotherapy regimen & \\
Fluorouracil $\left(425 \mathrm{mg} / \mathrm{m}^{2}\right) /$ Leucovorin $(100 \mathrm{mg})$ & $4(3.3)$ \\
One cycle & $8(6.6)$ \\
Two cycles & \\
Tegafur/Uracil & $1(0.8)$ \\
$200 \mathrm{mg} / 450 \mathrm{mg}$ per day & $1(0.8)$ \\
$300 \mathrm{mg} / 675 \mathrm{mg}$ per day & $102(84.3)$ \\
$400 \mathrm{mg} / 900 \mathrm{mg}$ per day & $1(0.8)$ \\
$600 \mathrm{mg} / 1,350 \mathrm{mg}$ per day & \\
Capecitabine & $2(1.6)$ \\
$2,000 \mathrm{mg}$ per day & $1(0.8)$ \\
$2,500 \mathrm{mg}$ per day & $1(0.8)$ \\
Miscellaneous usage &
\end{tabular}

"Tegafur/Uracil $400 \mathrm{mg} / 900 \mathrm{mg}$ then changed to Capecitabine 2,000 mg per day. ECE, extracapsular extension; ILR, intratumoral lymphocytic response; PLR, peritumoral lymphocytic response.

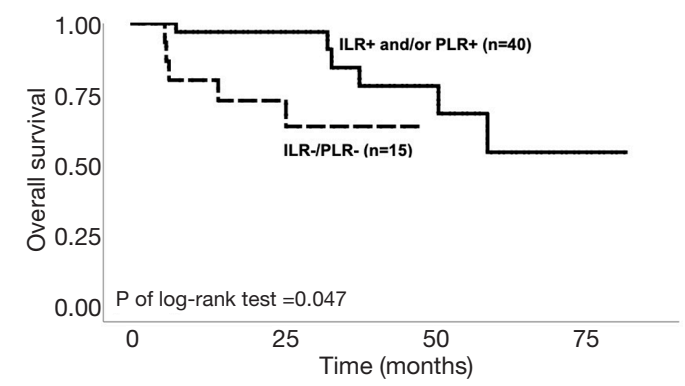

Figure 2 Overall survival of lymphocytic response

either intratumoral or peritumoral lymphocytic response. The hazard ratio of ILR-/PLR- were also significant as a risk factor of overall survival after adjusting pathological stage and margin status. Another significant risk factor was pathological lymph node stage.

Although the subgroups of lymphocytes cannot be discriminated on H\&E stain, it has been demonstrated that the T-cell cytotoxicity are stimulated in tumors with infiltrating lymphocytes (11). Lymphocytic infiltration has also been suggested as a prognostic variable in rectal cancer and other varieties of cancers $(12,13)$. The low density of immune biomarkers is associated with poor survival 
Table 2 Univariate and multivariate analyses integrating lymphocytic response and clinical characteristics

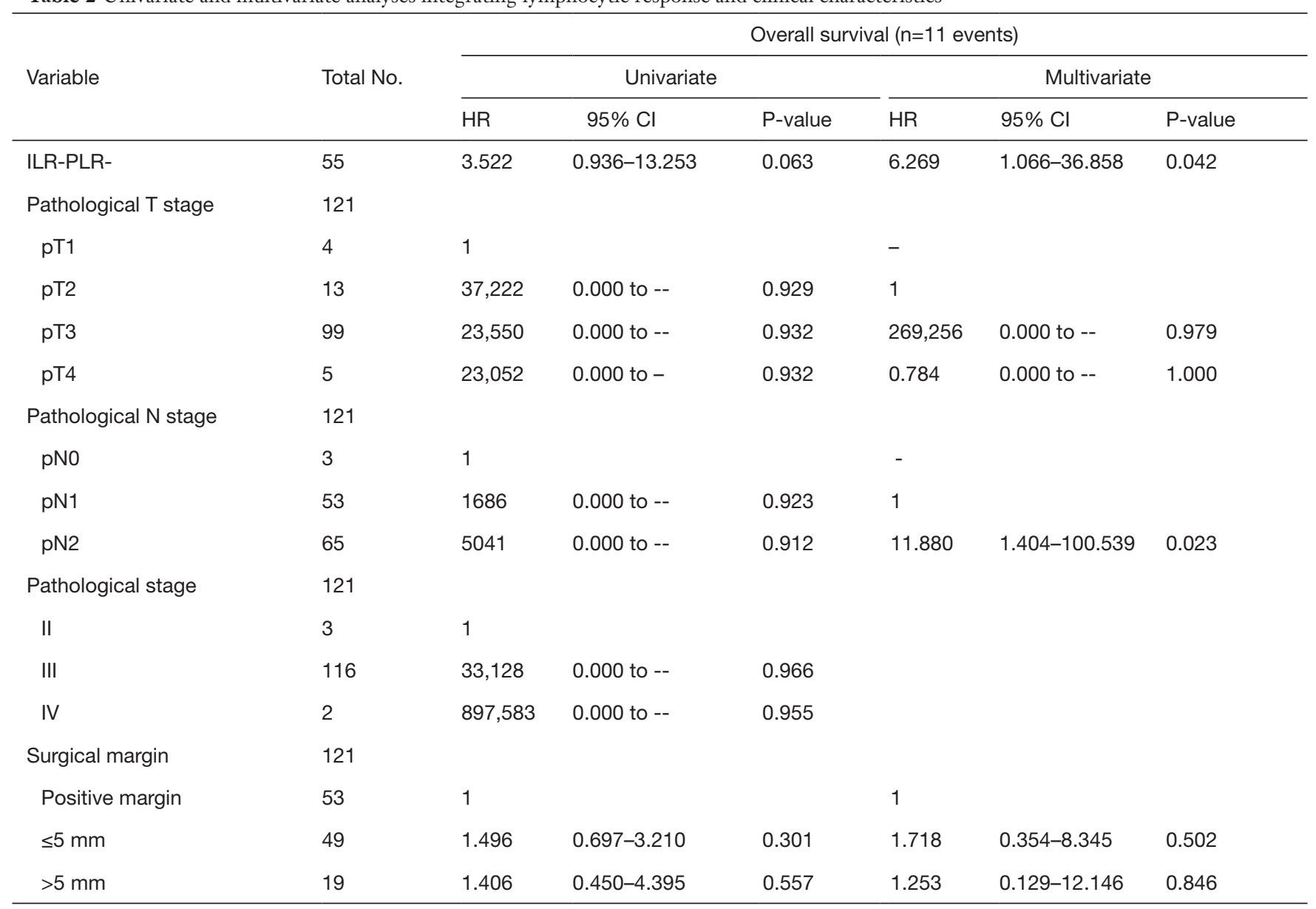

prognosis and may also increase the difficulty of treatment in colorectal cancer (14).

In our study, there is a trend of survival benefit on tumors with ILR+, which is consistent with the published evidence that there is a survival advantage of individuals with CRCs containing many TILs over those who has less TILs (15-18). The peritumoral lymphocytic response (PLR), or Crohn's disease-like reaction, is the lymphoid aggregation within the muscularis propria or pericolic adipose tissue, mostly located one or more millimeters beyond the advancing tumor fronts. Thus, PLR can be regarded as the lymphocytic response occurring in the tumor microenvironment (TME). There is evidence demonstrating the lack of Crohn's disease-like reaction indicates poor survival prognosis (19), supporting our result that PLR-/ILR- predicts poor survival outcome as well.

PLR has been correlated with a lower incidence of nodal metastasis in colorectal carcinoma $(8,19)$. In several published data, PLR is associated with improved cancerspecific survival and overall survival (20). To be noted, high PLR is also correlated with intense lymphocyte infiltration intratumorally and peritumorally $(19,21,22)$. CD $8+$ cytotoxic $\mathrm{T}$ lymphocyte is the most informative subset of lymphocytes to prognosis in anti-tumor immunity (23). Whether ILR and PLR are mainly consisted of CD8+ cytotoxic $\mathrm{T}$ lymphocytes remains to be determined by methods such as immunohistochemistry staining. The improved prognosis may be related to the up-regulated immune response and recruitment of lymphocyte targeting tumor cells, indicating an integrated perspective that combines ILR and PLR to predict prognosis. Adoptive cell therapy (ACT) using tumor-infiltrating lymphocytes (TIL) may be one of the potential clinical application and pharmaceutical meaning of ILR/PLR. ACT with TIL, which is based on infusion of in-vitro expanded autologous T-cells obtained from TME of the individual 
patients, has demonstrated curative potential in metastatic melanoma (24). The patients with the phenotype of immune-desert who presented by ILR-/PLR- in our study may be benefited from ACT with TIL in the future.

The limitation of our study is the relatively small sample size $(n=55)$. Another limitation is the lack of evidence that the amount of lymphocyte can reflect the activity of immune response. Thus, acquiring fresh specimen for biochemical analysis and the correlation between ILR/PLR and the effect of immunotherapy could be the direction of future studies.

For now, one of the application of ILR/PLR is to assist the decision making of rectal cancer treatment. According to our study, patients with ILR-/PLR- can be regarded as the phenotype of immune-desert and may not be a good candidate to receive immunotherapy taking the advantage of the interaction between tumor cells and peritumoral lymphocytes, though more studies needs to be explored. Instead, they may need intensified chemotherapy or radiotherapy.

To conclude, ILR-/PLR- can be a predictor for poor survival outcome and a potential biomarker for immune activity in torso. More cases and a molecular mechanism are needed for further investigation. This study provides a specific linkage for rectal cancer and the lymphocytic response in TME.

\section{Acknowledgments}

We would like to thank all members of the Department of Radiation Oncology, Department of Pathology and those who had collaborated to the patient treatments in this study. We also thank the editor and series editor for constructive criticisms of an earlier version of this chapter. This study was performed in accordance with ethical standards.

Funding: None.

\section{Footnote}

Reporting Checklist: The authors have completed the STROBE reporting checklist. Available at https://dx.doi. org/10.21037/tro-21-13

Conflicts of Interest: All authors have completed the ICMJE uniform disclosure form (available at https://dx.doi. org/10.21037/tro-21-13). Yu-Jen Chen serves as an Editorin-Chief of Therapeutic Radiology and Oncology. The other authors have no conflicts of interest to declare.
Ethical Statement: The authors are accountable for all aspects of the work in ensuring that questions related to the accuracy or integrity of any part of the work are appropriately investigated and resolved. The study was conducted in accordance with the Declaration of Helsinki (as revised in 2013). This study was approved by the local institutional review board of MacKay Memorial Hospital (IRB number: 20MMHIS005e; Date of decision: February $04,2020)$ and individual consent for this retrospective analysis was waived.

Open Access Statement: This is an Open Access article distributed in accordance with the Creative Commons Attribution-NonCommercial-NoDerivs 4.0 International License (CC BY-NC-ND 4.0), which permits the noncommercial replication and distribution of the article with the strict proviso that no changes or edits are made and the original work is properly cited (including links to both the formal publication through the relevant DOI and the license). See: https://creativecommons.org/licenses/by-nc-nd/4.0/.

\section{References}

1. Bray F, Ferlay J, Soerjomataram I, et al. Global cancer statistics 2018: GLOBOCAN estimates of incidence and mortality worldwide for 36 cancers in 185 countries. CA Cancer J Clin 2018;68:394-424.

2. Samowitz WS, Curtin K, Ma KN, et al. Microsatellite instability in sporadic colon cancer is associated with an improved prognosis at the population level. Cancer Epidemiol Biomarkers Prev 2001;10:917-23.

3. Mei Z, Liu Y, Liu C, et al. Tumour-infiltrating inflammation and prognosis in colorectal cancer: systematic review and meta-analysis. Br J Cancer 2014;110:1595-605.

4. Elsaleh H, Joseph D, Grieu F, et al. Association of tumour site and sex with survival benefit from adjuvant chemotherapy in colorectal cancer. Lancet 2000;355:1745-50.

5. Toor SM, Sasidharan Nair V, Murshed K, et al. TumorInfiltrating Lymphoid Cells in Colorectal Cancer Patients with Varying Disease Stages and Microsatellite InstabilityHigh/Stable Tumors. Vaccines 2021;9:64.

6. Koi M, Carethers JM. The colorectal cancer immune microenvironment and approach to immunotherapies. Future Oncol 2017;13:1633-47.

7. Mlynska A, Vaisnore R, Rafanavicius V, et al. A gene signature for immune subtyping of desert, excluded, 
and inflamed ovarian tumors. Am J Reprod Immunol 2020;84:e13244.

8. Rozek LS, Schmit SL, Greenson JK, et al. TumorInfiltrating Lymphocytes, Crohn's-Like Lymphoid Reaction, and Survival From Colorectal Cancer. J Natl Cancer Inst 2016;108:djw027.

9. Ceelen W, Fierens K, Van Nieuwenhove Y, et al. Preoperative chemoradiation versus radiation alone for stage II and III resectable rectal cancer: a systematic review and meta-analysis. Int J Cancer 2009;124:2966-72.

10. Alexander J, Watanabe T, Wu T-T, et al. Histopathological Identification of Colon Cancer with Microsatellite Instability. Am J Pathol 2001;158:527-35.

11. Dolcetti R, Viel A, Doglioni C, et al. High prevalence of activated intraepithelial cytotoxic $\mathrm{T}$ lymphocytes and increased neoplastic cell apoptosis in colorectal carcinomas with microsatellite instability. Am J Pathol 1999;154:1805-13.

12. Jass JR. Lymphocytic infiltration and survival in rectal cancer. J Clin Pathol 1986;39:585-9.

13. Gooden MJ, de Bock GH, Leffers N, et al. The prognostic influence of tumour-infiltrating lymphocytes in cancer: a systematic review with meta-analysis. Br J Cancer 2011;105:93-103.

14. Craig SG, Humphries MP, Alderdice M, et al. Immune status is prognostic for poor survival in colorectal cancer patients and is associated with tumour hypoxia. $\mathrm{Br} \mathrm{J}$ Cancer 2020;123:1280-8.

15. Svennevig JL, Lunde OC, Holter J, et al. Lymphoid infiltration and prognosis in colorectal carcinoma. $\mathrm{Br} \mathrm{J}$ Cancer 1984;49:375-7.

16. Huh JW, Lee JH, Kim HR. Prognostic Significance

doi: $10.21037 /$ tro-21-13

Cite this article as: Su CW, Tang TY, Li CJ, Huang YC, Chen YJ. Intratumoral and peritumoral lymphocytic responses correlate with survival in rectal cancer. Ther Radiol Oncol 2021;5:14. of Tumor-Infiltrating Lymphocytes for Patients With Colorectal Cancer. Arch Surg 2012;147:366-72.

17. Galon J, Costes A, Sanchez-Cabo F, et al. Type, density, and location of immune cells within human colorectal tumors predict clinical outcome. Science 2006;313:1960-4.

18. Nosho K, Baba Y, Tanaka N, et al. Tumour-infiltrating T-cell subsets, molecular changes in colorectal cancer, and prognosis: cohort study and literature review. J Pathol 2010;222:350-66.

19. Harrison JC, Dean PJ, El-zeky F, et al. Impact of the Crohn's-like lymphoid reaction on staging of right-sided colon cancer: Results of multivariate analysis. Hum Pathol 1995;26:31-8.

20. Ogino S, Nosho K, Irahara N, et al. Lymphocytic reaction to colorectal cancer is associated with longer survival, independent of lymph node count, microsatellite instability, and $\mathrm{CpG}$ island methylator phenotype. Clin Cancer Res 2009;15:6412-20.

21. Graham DM, Appelman HD. Crohn's-like lymphoid reaction and colorectal carcinoma: a potential histologic prognosticator. Mod Pathol 1990;3:332-5.

22. Väyrynen JP, Sajanti SA, Klintrup K, et al. Characteristics and significance of colorectal cancer associated lymphoid reaction. Int J Cancer 2014;134:2126-35.

23. Farhood B, Najafi M, Mortezaee K. CD8(+) cytotoxic T lymphocytes in cancer immunotherapy: A review. J Cell Physiol 2019;234:8509-21.

24. Forget MA, Haymaker C, Hess KR, et al. Prospective Analysis of Adoptive TIL Therapy in Patients with Metastatic Melanoma: Response, Impact of Anti-CTLA4, and Biomarkers to Predict Clinical Outcome. Clin Cancer Res 2018;24:4416-28. 